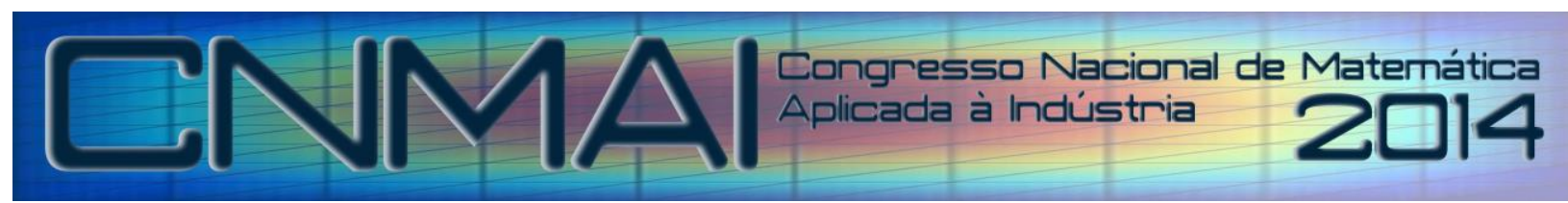

18 a 21 de novembro de 2014, Caldas Novas - Goiás

\title{
Técnicas de sequenciamento para o auxílio em execuções de projetos automobilísticos
}

\author{
Tiago dos Santos Almeida, tiago_dosalmeida@ hotmail.com ${ }^{1}$ \\ Helio Yochihiro Fuchigami, heliofuchigami@yahoo.com.br ${ }^{2}$ \\ ${ }^{1}$ Universidade Federal de Goiás- Departamento de Matemática Industrial, Av. Dr. Lamartine Pinto de \\ Avelar, 1120, Setor Universitário. Bolsista FAPEG. \\ ${ }^{2}$ Universidade Federal de Goiás- Departamento de Matemática Industrial, Av. Dr. Lamartine Pinto de \\ Avelar, 1120, Setor Universitário
}

Resumo: Dentre as dificuldades apresentadas no decorrer de uma execução de projetos, o tempo tem sido um dos fatores mais importantes para o sucesso. Ocorre que na fase de planejamento, dedica-se um período considerável para preparar e definir cada fase do projeto, além de estimar as durações de cada atividade. O objetivo deste trabalho é demonstrar a importância que métodos de sequenciamento possuem para a execução de projetos em empresas. Os procedimentos para a elaboração deste trabalho consistiram em uma revisão bibliográfica das técnicas de sequenciamento, conceitos e riscos nas execuções de projetos. Realizando um estudo de caso em um departamento participante de projetos automobilísticos.

Palavras-chave: Sequenciamento. Projeto. Tarefas.

\section{INTRODUÇÃO}

Dentre as dificuldades apresentadas no decorrer de uma execução de projetos, o tempo tem sido um dos fatores mais importantes para o sucesso. Ocorre que na fase de planejamento, dedica-se um período considerável para preparar e definir cada fase do projeto, além de estimar as durações de cada atividade. Entretanto, por mais que um projeto esteja bem planejado, o mesmo será influenciado por diversos fatores que caso não forem bem gerenciados comprometeram o sucesso do mesmo.

Para Valeriano (1998) apud Moreira et.al.(2010 dentre das áreas de conhecimento de projetos que deve ter um gerenciamento mais rígido, é o tempo; sua administração está diretamente ligada ao sincronismo das atividades participantes no projeto. Portanto, para que o mesmo possa ser concluído no tempo previsto é imprescindível se fazer um minucioso controle e acompanhamento de todas essas atividades com a elaboração de um cronograma.

Apesar das grandes experiências das equipes de planejamento de um projeto, os valores que são definidos como tolerâncias para a conclusão de cada atividade, poderão fugir do controle caso os riscos não forem avaliados e definidos soluções para o caso de ocorrência, resultando em grandes atrasos nas entregas.

O risco de cada projeto é sempre futuro. O risco é uma fase ou uma esfera incerta, que se caso ocorrer, tem um efeito no mínimo em um objetivo do projeto. Os objetivos que podem incluir escopo, cronograma, qualidade e custo (PMBOK, 2008).

Moreira (2010) salienta que o tempo está diretamente associado aos três fatores seguintes: escopo, custo e qualidade; portanto, qualquer alteração em um deles, os demais podem ser afetados, por exemplo, qualquer atraso em seu tempo de um projeto poderá resultar na mudança de escopo, alteração da planta, entre outras. 
As dificuldades encontradas no decorrer das execuções de cada atividade planejada, em diversas ocasiões são influencias por fatores de uma má escolha do recurso, ou até mesmo de uma falta de estrutura na equipe de execução. A equipe técnica, responsável em executar as atividades, deverá ser instruída de todos os riscos que um projeto possui, buscando fornecer métodos e soluções para gerenciá-las. Sendo assim, este trabalho abordará aspectos ligados aos riscos de um mau gerenciamento do tempo, buscando apresentar técnicas que auxiliem a equipe do projeto.

O objetivo deste trabalho é demonstrar a importância que métodos de sequenciamento possuem para a execução de projetos em empresas em que há o desenvolvimento simultâneo de projetos, além de contribuir com técnicas para o gerenciamento do tempo. Será realizado um estudo de caso em uma empresa automobilística, situada no estado de Goiás.

Os procedimentos para a elaboração deste trabalho consistiu em uma revisão bibliográfica das técnicas de sequenciamento, conceitos e riscos nas execuções de projetos. Realizando um estudo de caso em um departamento participante de projetos automobilísticos.

\section{REVISÃO BIBLIGRÁFICA}

\subsection{Projetos}

Conforme Vargas (2005), projeto é um empreendimento não constante, caracterizado por uma sequência clara e lógica de eventos, tendo definido um início, meio e fim, que tem meta atingir a um objetivo claro e definido, sendo conduzido por pessoas segundo parâmetros predefinidos de custo, tempo, qualidade e recursos envolvidos.

As principais áreas de aplicação de projetos são expostas por Vagas (2005) da seguinte maneira:

- Engenharia Civil;

- Desenvolvimento de programas de computador;

- Estratégia militar;

- Administração de empresas;

- Marketing e publicidade;

- Pesquisa e desenvolvimento;

- Manutenção de planta e equipamentos.

\subsection{Riscos}

Segundo estudo de Pinto (2009), diversos fatores foram analisados com o intuito de expor o grau de ocorrência em projetos. Dentre os problemas mais encontrados em um projeto, o requisito de não cumprimento dos prazos, têm sido um dos mais ocorrentes, resultando em $71 \%$ de ocorrência. A figura 1 representa os problemas ocorridos nos projetos.

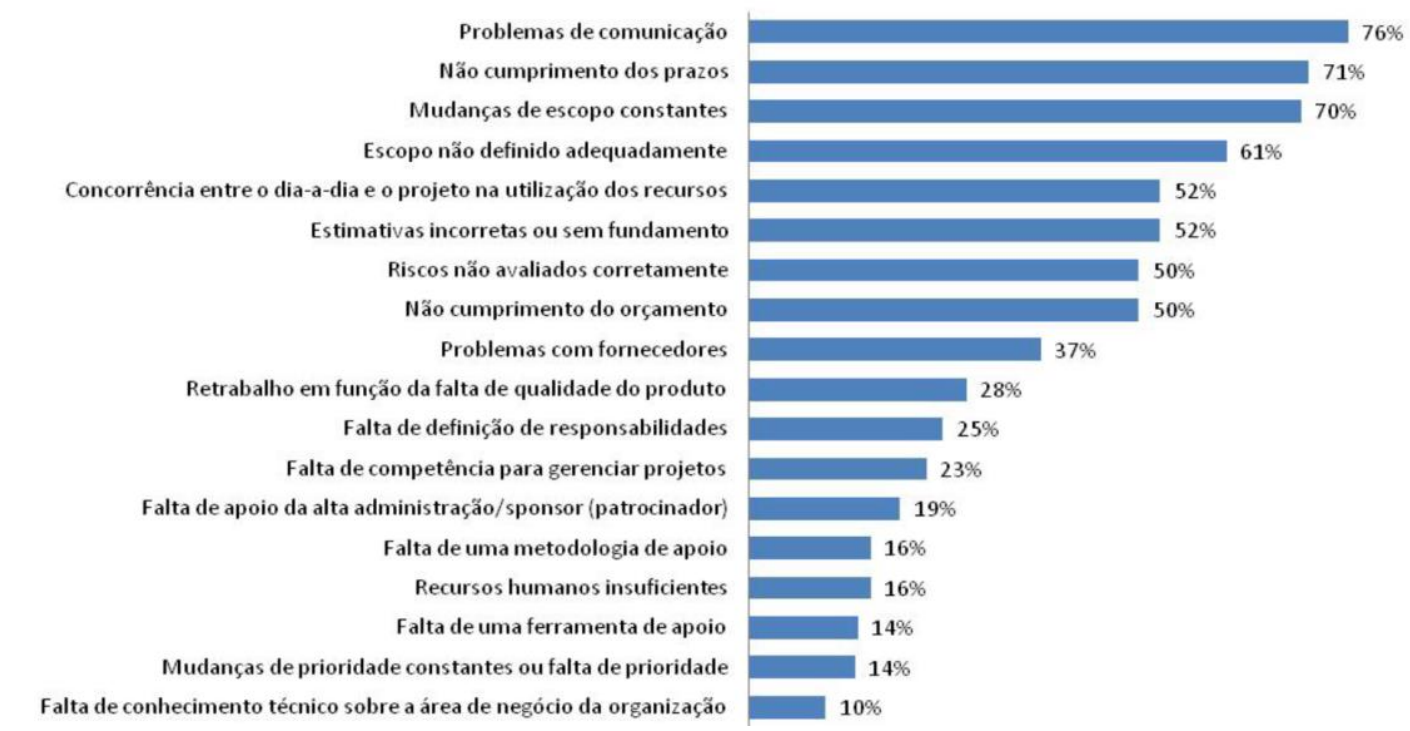

\subsection{Regras de sequenciamento}

Figura 1. Principais problemas em projetos. Fonte: Pinto (2009) 
Silva et al. (2012) exemplificam as principais regras de sequenciamento com base nos autores: Mesquita et al. (2008), Tubino (2007), Suresh e Sridharan (2007), Chan e Chan, (2004) e Gaither e Frazier (2002).

- SPT - (Shortest Processing Time), a prioridade é dada pelo menor tempo de processamento total. É classificada em ordem crescente de tempo. Sua utilização visa reduzir o tamanho das filas e o aumento do fluxo.

- LPT - (Longest Processing Time), a prioridade é dada pelo maior tempo de processamento total. Contrário à regra SPT. Sua utilização visa à redução de troca de máquinas.

- EDD - (Earliest Due Date), a prioridade é dada pela execução das ordens mais urgentes em termos de prazo de entrega. $\mathrm{O}$ foco é reduzir atrasos.

\section{ESTUDO DE CASO}

\subsection{Histórico}

As atividades da empresa foram iniciadas em setembro de 1998, quando a marca decidiu se instalar no estado de Goiás. Esta decisão foi resultado da abertura comercial brasileira às importações no ano de 1990, onde na cidade de São Paulo foi inaugurada uma central de vendas. Com o sucesso do produto, o mercado de concessionária se expandiu por todo o Brasil, que ao final de dois anos resultou-se em 20 pontos de vendas.

Sendo uma empresa com capital 100\% nacional, com o seu sucesso, em 1998 foi iniciada uma nova fase na história da empresa. A decisão de instalar uma montadora no estado de Goiás apresentou no ano de 2007 a vitoriosa marca de 100 mil veículos produzidos.

Com o sucesso da marca, o um projeto de ampliação foi o próximo grande passo no Brasil. O que tornou o espaço de $14 \mathrm{mil} \mathrm{m}^{2}$ de área construída para $57 \mathrm{mil} \mathrm{m}^{2}$. Assim, o número de linhas de produção passou de 2 para 3 e praticamente dobrou a capacidade de produção anual, de 15 mil unidades para 28 mil. Atualmente com a produção de novos veículos, a fábrica está com a capacidade de produção de 100 mil carros/ano.

\subsection{Modelagem do problema}

Com o intuito de utilizar regras de sequenciamento para o auxílio nas execuções de atividades de um projeto, em função das dificuldades apresentadas por participantes do projeto para as entregas das atividades, será realizada uma série de testes de sequenciamento com as atividades inerentes do projeto.

Desta forma, será destacado um grupo de atividades que norteiam a fase de planejamento logístico de um projeto em uma empresa automobilística. Serão descritos na tabela abaixo, 10 atividades que consistem em uma análise, distribuição e definições detalhadas, com base em folhas de processos, no intuito de identificar as necessidades de investimento para um novo veículo.

Tabela 1. Grupo de atividades de um projeto.

\begin{tabular}{|c|c|c|}
\hline ITEM & ATIVIDADE & DURAÇÃO (DIAS) \\
\hline$\ldots$. & .... & $\ldots$ \\
\hline 45 & Distribuição estimada de embalagens específicas por item & 20 \\
\hline 46 & Distribuição estimada de embalagens padrão por item (cesto, 1/2 GG, base GG, caixas plásticas P, M, G e EG) & 20 \\
\hline 47 & Distribuição estimada de método de abastecimento por item & 10 \\
\hline 48 & Estimativa de quantidade de estantes flow racks & 3 \\
\hline 49 & Distribuição estimada por item de embalagens nacionais no line feeding & 15 \\
\hline 50 & Distribuição estimada de bases necessárias para transporte de componentes nacionais & 3 \\
\hline 51 & Distribuição estimada de itens sequenciados entre as plataformas ZC e GS41 & 20 \\
\hline 52 & Estimativa e distribuição de itens que podem ser abastecidos em supermercado para linha de cabine & 20 \\
\hline 53 & Estimativa de verba necessário & 3 \\
\hline 54 & Aprovação do memorial de embalagens (MARCO) & 1 \\
\hline$\ldots$. & $\ldots$. & $\ldots$. \\
\hline
\end{tabular}

Na Tabela 2 é representada uma fase do projeto em que há uma série de atividades atrasadas e que há um comprometimento da data final da fase de planejamento. Dentre as 10 atividades apresentadas, 5 atividades permanecem atrasadas.

Tabela 2. Atividades atrasadas e concluídas. 


\begin{tabular}{|c|c|c|c|c|c|c|}
\hline 5 & Distribuição estimada de embalagens específicas por item & 20 dias & Sex 07/06/13 & Qui 04/07/13 & $100 \%$ & Concluída \\
\hline$\Rightarrow$ & $\begin{array}{l}\text { Distribuição estimada de embalagens padrão por item (cesto, } 1 / 2 \\
\text { GG, base } G G \text {, caixas plásticas } P, M, G \text { e EG) }\end{array}$ & 20 dias & $\operatorname{Sex} 07 / 06 / 13$ & Qui 04/07/13 & $100 \%$ & Concluída \\
\hline 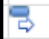 & Distribuição estimada de método de abastecimento por item & 10 dias & Sex $05 / 07 / 13$ & Qui $18 / 07 / 13$ & $85 \%$ & Atrasada \\
\hline 8 & Estimativa de quantidade de estantes flow racks & 5 dias & Sex $12 / 07 / 13$ & Qui $18 / 07 / 13$ & $100 \%$ & Concluída \\
\hline 8 & $\begin{array}{l}\text { Distribuição estimada por item de embalagens nacionais no line } \\
\text { feeding }\end{array}$ & 23 dias & Sex $12 / 07 / 13$ & Ter $13 / 08 / 13$ & $100 \%$ & Concluída \\
\hline 8 & $\begin{array}{l}\text { Distribuição estimada de bases necessárias para transporte de } \\
\text { componentes nacionais }\end{array}$ & 15 dias & Qua $24 / 07 / 13$ & Ter $13 / 08 / 13$ & $100 \%$ & Concluída \\
\hline$\Rightarrow$ & $\begin{array}{l}\text { Distribuição estimada de itens sequenciados entre as plataformas ZC } \\
\text { e GS41 }\end{array}$ & 10 dias & $\operatorname{Sex} 12 / 07 / 13$ & Qui $25 / 07 / 13$ & $50 \%$ & Atrasada \\
\hline 8 & $\begin{array}{l}\text { Estimativa e distribuição de itens que podem ser abastecidos em } \\
\text { supermercado para linha de cabine }\end{array}$ & 10 dias & Sex $12 / 07 / 13$ & Qui $25 / 07 / 13$ & $50 \%$ & Atrasada \\
\hline 8 & Estimativa de verba necessário & 3 dias & Sex $26 / 07 / 13$ & Sex $16 / 08 / 13$ & $25 \%$ & Atrasada \\
\hline 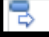 & Aprovação do memorial de embalagens (MARCO) & 0 dias & Sex $16 / 08 / 13$ & Sex $16 / 08 / 13$ & $0 \%$ & Atrasada \\
\hline
\end{tabular}

Para as execuções deste grupo de atividades, é utilizado um único recurso. Portanto, propõe-se que seja utilizada uma regra que minimize o número de tarefas atrasadas.

\subsection{Método de solução proposto}

Como método de solução para o problema proposto, serão utilizadas as seguintes regras de sequenciamento: SPT, LPT e EDD. Para a realização das experimentações, será utilizado o software Lekin, para o apoio deste estudo.

\section{ANÁLISE DE DADOS}

Para a elaboração das experimentações das técnicas de sequenciamento, utilizaram-se os dados da Tabela 3:

Tabela 3: Duração das atividades (Pj), datas de liberação ( $R \mathbf{j})$ e prazos (Dj).

\begin{tabular}{|c|c|c|c|c|c|c|c|c|c|c|}
\cline { 2 - 11 } \multicolumn{1}{c|}{} & $\mathbf{J}_{\mathbf{1}}$ & $\mathbf{J}_{\mathbf{2}}$ & $\mathbf{J}_{\mathbf{3}}$ & $\mathbf{J}_{\mathbf{4}}$ & $\mathbf{J}_{\mathbf{5}}$ & $\mathbf{J}_{\mathbf{6}}$ & $\mathbf{J}_{\mathbf{7}}$ & $\mathbf{J}_{\mathbf{3}}$ & $\mathbf{J}_{\mathbf{9}}$ & $\mathbf{J}_{\mathbf{1 0}}$ \\
\hline $\mathbf{P}_{\mathbf{j}}$ & 20 & 20 & 10 & 3 & 15 & 3 & 20 & 20 & 3 & 1 \\
\hline $\mathrm{R}_{\mathbf{j}}$ & 0 & 0 & 29 & 36 & 36 & 46 & 36 & 36 & 48 & 40 \\
\hline $\mathrm{D}_{\mathbf{j}}$ & 20 & 20 & 39 & 39 & 51 & 49 & 56 & 56 & 51 & 41 \\
\hline
\end{tabular}

\subsection{Medidas de desempenho}

Com base nos testes realizados, foi realizada uma série de análises com base nos indicadores fornecidos pelo software Lekin, que podem ser compreendidos como:

- Makespan: é o tempo de duração das 10 atividades, no qual pode ser compreendido o tempo despendido para a conclusão das mesmas.

- Max. Tardiness: é o máximo de tempo de atraso das atividades.

- Number of late Jobs: é o número de tarefas atrasadas.

- Total Flow Time: é o tempo total de fluxo nas execuções de cada atividade.

- Total Tardiness: é o tempo total de atraso das atividades.

Sendo assim, para uma análise detalhada de cada uma das regras de seqüenciamento, devem-se compreender os principais objetivos do projeto. No caso do projeto utilizado para a realização de testes deste trabalho, o principal foco é atender as datas de entregas de cada atividade, de tal maneira a garantir que as metas sejam cumpridas, ou seja, eliminar todos os possíveis atrasos nas execuções de cada atividade. Essa metodologia é aplicada, em função de ter uma série de atividades que influenciam nas execuções de outros recursos, que só serão iniciadas com as conclusões de outras atividades dependentes. Como resultado final, a SPT obteve 5 atividades atrasadas, que diante da política da empresa, garantiu o bom andamento deste projeto, que em relação aos demais testes utilizados, gerou um impacto de atraso menor.

\subsection{Regra SPT}

Os valores apresentados na duração das 10 atividades foram de 127 dias, com o número de 5 atividades atrasadas. A figura 2 está representando o gráfico de Gantt da regra testada. 


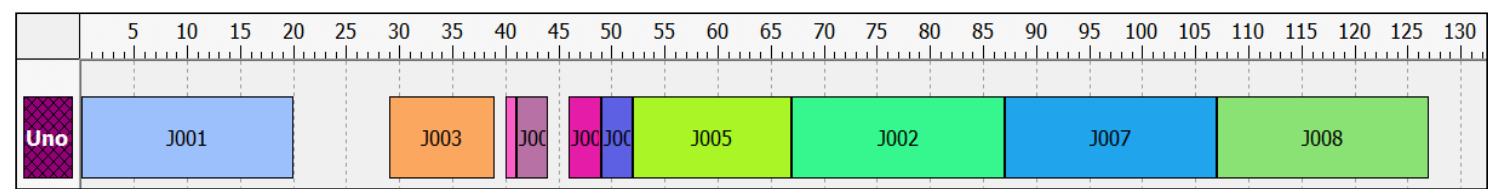

Figura 2. Gráfico de Gantt para a regra SPT.

A figura 3 representa os indicadores de desempenho da regra SPT.

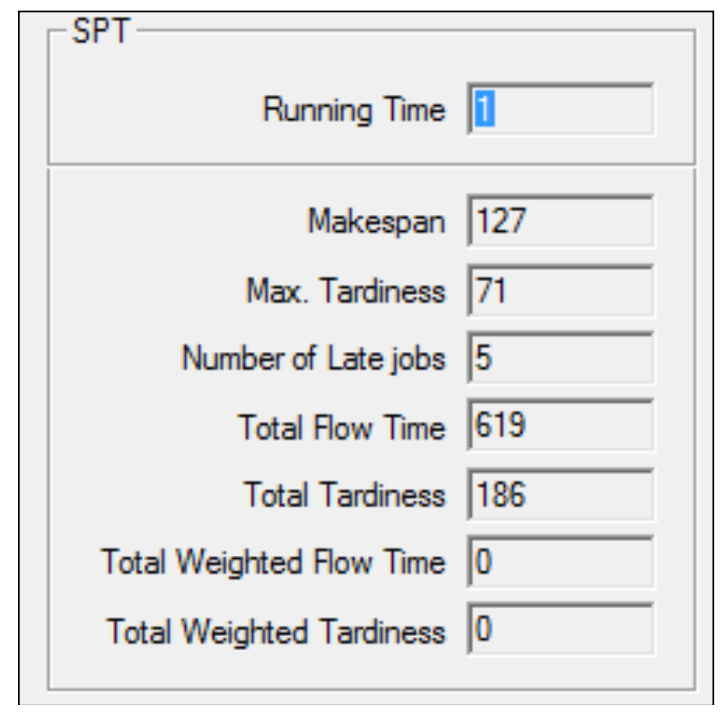

Figura 3. Indicadores de desempenho da regra SPT.

\subsection{Regra SPT}

Os valores apresentados na duração das 10 atividades foram de 115 dias, com o número de 8 atividades atrasadas. A figura 4 está representando o gráfico de Gantt da regra testada.

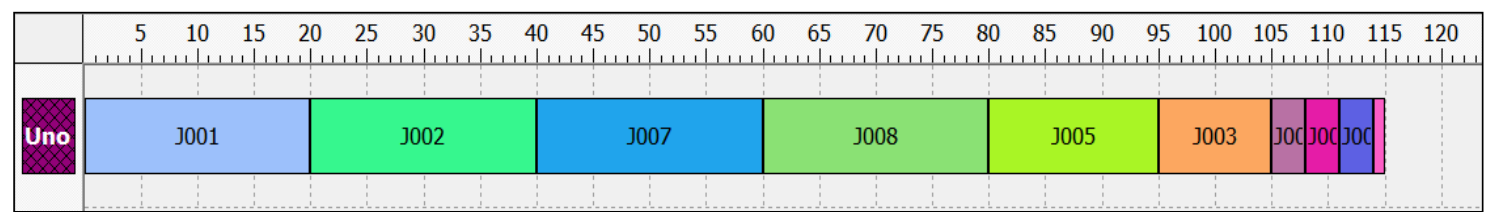

Figura 4. Gráfico de Gantt para a regra LPT.

A figura 5 representa os indicadores de desempenho da regra LPT.

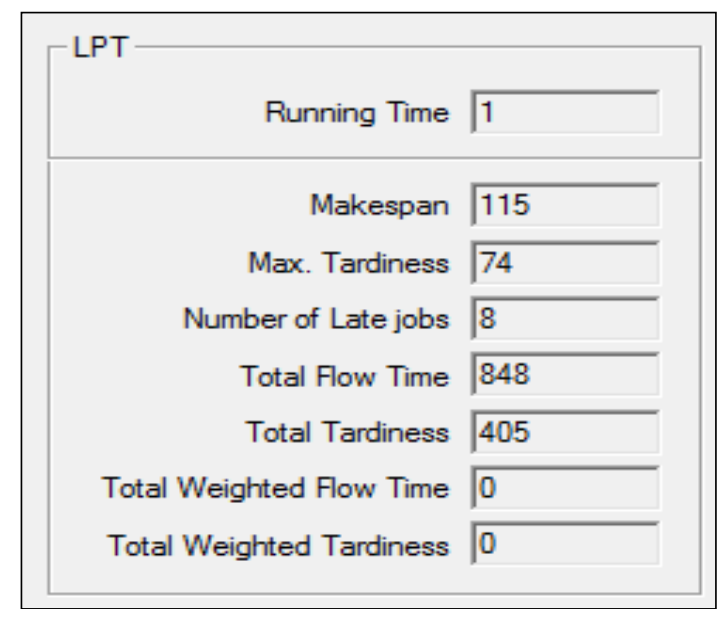

Figura 5. Indicadores de desempenho da regra LPT. 


\subsection{Regra EDD}

Os valores apresentados na duração das 10 atividades foram de 115 dias, com o número de 8 atividades atrasadas. A figura 6 está representando o gráfico de Gantt da regra testada.

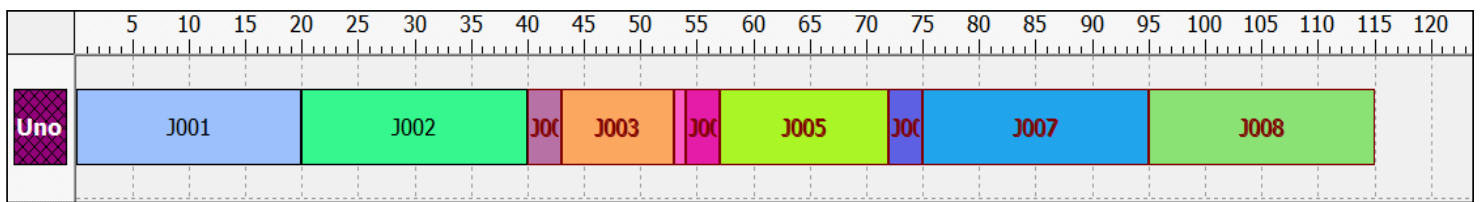

Figura 6. Gráfico de Gantt para a regra EDD.

A figura 7 representa os indicadores de desempenho da regra EDD.

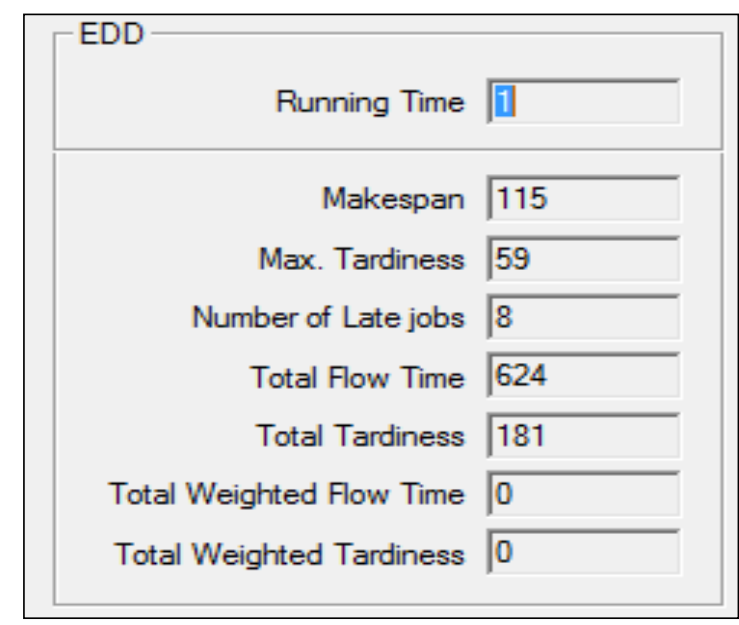

Figura 7. Indicadores de desempenho da regra EDD.

\section{CONCLUSÃO}

Com base nas experimentações realizadas através do software Lekin, a regra que possibilitou menor quantidade de tarefas atrasadas foi a SPT (shortest processing time), no total 5 atividades. Desta forma, o objetivo deste trabalho foi alcançado, em virtude de ter sido experimentado 3 tipos de regras de sequenciamento.

A proposta de alinhar as metodologias de sequenciamento alinhado com as execuções das atividades de um projeto é em função da necessidade de maior orientação por parte dos recursos utilizados, bem como no alcance dos objetivos principais com cada entrega no tempo correto das atividades. É importante salientar que para cada tipo de projeto deve ser compreendido seu objetivo geral, bem como o seu funcionamento, levando em consideração as correlações de cada atividade, com o propósito de identificar qual regra de sequenciamento se adequará melhor. No projeto utilizado, a regra SPT atendeu melhor os objetivos do projeto, em virtude da não aceitação por parte do gestor nos atrasos das atividades, pois ao final do projeto, poderia prejudicar nas execuções de outras pessoas, com a possibilidade comprometer a implementação de um novo veículo.

Este trabalho contribui para divulgar as metodologias de sequenciamento nas execuções de atividades de projetos automobilísticos, além de estender às outras áreas.

\section{REFERÊNCIAS}

Valeriano, D. L. 1998. Gerência em projetos: pesquisa, desenvolvimento e engenharia. São Paulo: Makron Books.

Moreira, A.M., Silva, R.S., Palma, M.A.M. 2010. Revista de Gestão e Projetos - GeP, São Paulo, v. 1, n. 2, p 128-146, jul./dez.

Silva, E. B. et al. 2012. Avaliação de regras de sequenciamento da produção em ambientes Job shop e Flow shop por meio de simulação computacional. Exacta, vol. 10, núm. 1, enero-junio, pp. 70-81, Universidade Nove de Julho Brasil. 
PMBOK, Um guia do conhecimento em gerenciamento de projetos (Guia PMBOK). $4^{\mathrm{a}}$ edição, 2008 Project Management Institute, Inc.

Pinto, A. 2009. Relatório principal do estudo de benchmarking: gerenciamento de projetos Brasil. Biblioteca do site da Manager Brazil: Consultoria e Treinamento em Projetos. Estudo de Benchmarking em Gerenciamento de Projetos Brasil, Project Management Institute - Chapters Brasileiros.

Vargas, V., R. 2005. Gerenciamento de Projetos: estabelecendo diferenciais competitivos / Ricardo Viana Vargas: prefácio de Reeve Harold R - 6.ed.atual. - Rio de janeiro - Brasport.

Silva, E.B.; Costa, M.G.; Souza Silva. M.F. Pereira. F.H. 2012. Avaliação de regras de sequenciamento da produção em ambientes Job shop e Flow shop por meio de simulação computacional. Exacta, vol. 10, núm. 1, enero-junio, pp. 70-81, Universidade Nove de Julho Brasil. 


\title{
Sequencing techniques to aid in executions of automotive design
}

Tiago dos Santos Almeida, tiago_dosalmeida@hotmail.com ${ }^{1}$

Helio Yochihiro Fuchigami, heliofuchigami@yahoo.com.br ${ }^{2}$

${ }^{1}$ Federal University of Goiás- Department of Industrial Mathematics, Av. Dr. Lamartine Pinto de Avelar, 1120, University Sector. Scholarship from FAPEG.

${ }^{2}$ Federal University of Goiás- Department of Industrial Mathematics, Av. Dr. Lamartine Pinto de Avelar, 1120, University Sector

\begin{abstract}
Among the difficulties encountered in the course of a project execution time has been one of the most important factors for success. That occurs in the planning phase, dedicated considerable time to prepare and define each phase of the project period, and estimating the duration of each activity. The objective of this work is to demonstrate the importance of sequencing methods have to execute projects in companies. The procedures for the preparation of this work consisted of a literature review of sequencing techniques, concepts and risks in executing projects. Conducting a case study at a participating department of automotive design.
\end{abstract}

Palavras-chave: Sequencing. Project. Tasks. 